\title{
What Should We Expect From Research Through Design?
}

\author{
William Gaver \\ Interaction Research Studio \\ Goldsmiths, University of London \\ w.gaver@gold.ac.uk
}

\begin{abstract}
In this essay, I explore several facets of research through design in order to contribute to discussions about how the approach should develop. The essay has three parts. In the first, I review two influential theories from the Philosophy of Science to help reflect on the nature of design theory, concluding that research through design is likely to produce theories that are provisional, contingent, and aspirational. In the second part, I discuss three possible interpretations for the diversity of approaches to research through design, and suggest that this variation need not be seen as a sign of inadequate standards or a lack of cumulative progress in the field, but may be natural for a generative endeavour. In the final section, I suggest that, rather than aiming to develop increasingly comprehensive theories of design, practice based research might better view theory as annotation of realised design examples, and particularly portfolios of related pieces. Overall, I suggest that the design research community should be wary of impulses towards convergence and standardisation, and instead take pride in its aptitude for exploring and speculating, particularising and diversifying, and - especially - its ability to manifest the results in the form of new, conceptually rich artefacts.
\end{abstract}

\section{Author Keywords}

research through design; theory; annotation; portfolios; philosophy of science

\section{ACM Classification Keywords}

H.5.m [Information interfaces and presentation]: Miscellaneous - Design;

\section{INTRODUCTION}

Over the last number of years, design practitioners have become increasingly integrated within the HCI research community. Their work often takes the form of research through design [4, 11, 34], in which design practice is brought to bear on situations chosen for their topical and

Author Accepted Manuscript (AAM)

To appear in CHI'12, May 5-10, 2012, Austin, Texas, USA. theoretical potential, the resulting designs are seen as embodying designers' judgments about valid ways to address the possibilities and problems implicit in such situations, and reflection on these results allow a range of topical, procedural, pragmatic and conceptual insights to be articulated. The output of this work takes the form, primarily, of artefacts and systems, sometimes with associated accounts of how these are used in field tests, but increasingly includes a variety of methods, conceptual frameworks and theories presented separately from accounts of practice.

As design practice has become more prevalent in $\mathrm{CHI}$, however, there has also grown an undercurrent of questioning within the design community itself about the nature and standards of research through design $[9,31,33$, 35]. These discussions revolve around the desirability of 'integrating design research methods, approaches, and outcomes in HCI' [9] both as a way of consolidating knowledge and to establish criteria for research done from this perspective. This agenda calls for the development of agreed methodological standards, hand in hand with a firm theoretical foundation. Research through design, it is said, 'lacks clear expectations and standards for what constitutes "good" design research', and thus would benefit from 'some actionable metrics for bringing rigor in critique of design research' ([9] p824). The community should 'develop protocols, descriptions, and guidelines for its processes, procedures, and activities' in ways assumed to be 'like any other research approach' ([35] p317). In part, this depends on developing research through design as 'a proper research methodology that can produce relevant and rigorous theory' ([35] p316). Conceptual contributions, from this perspective, should exhibit 'a level of extensibility and verifiability' if they are to comprise 'theory designers can apply in research in practice' [33].

As a long-term practitioner of research through design, I have some sympathy with these concerns. Nonetheless, I am uneasy about some of the tendencies that seem to underlie both the diagnoses and the treatments that have been suggested. Seeking conformance to agreed-upon standards and processes may be a route towards disciplinary legitimacy within HCI, helping clarify expectations for research through design both within and outside the design community. There is a risk, however, that such standards might lead to a form of self-policing that would be overly restrictive of a form of research that I 
value for its ability to continually and creatively challenge status quo thinking. Moreover, insofar as $\mathrm{HCI}$ is prone to 'scientism' in its cultural assumptions (despite moves to legitimise other forms of research based, for instance, on the humanities [2], or to characterize it as a design discipline [7]), attempts to establish standards for research through design may adopt, or be interpreted in terms of, inappropriate 'scientific' models of research and theory for the field.

In this essay, my intention is to temper calls for disciplinary consolidation with remarks on what we might reasonably expect from research through design. First, I explore the kinds of theory that have been developed in association with design using two accounts of scientific theory drawn from the Philosophy of Science. Contrasting accounts are chosen to complicate notions of what makes theories 'scientific', in order to undermine any overly simple assumptions about how research should proceed, and to draw out fundamental features of the conceptual work that accompanies research through design. This comparison suggests that, instead of being extensible and verifiable, theory produced by research through design tends to be provisional, contingent and aspirational. Second, calls for standards, formalisation, protocols and guidelines assume that disciplinary convergence is a prerequisite for cumulative growth of understanding in design. I explore three perspectives on the lack of convergence in research through design, and suggest that, while one of these perspectives implies that the community should work towards greater shared understandings, the other two imply either that there is no problem to solve or that progress in this area will be marked by proliferation rather than agreement. Third, an emphasis on agreed-upon theories and methods seems to assume that these are the hallmark of a respectable research discipline, and that the production of an endless stream of design examples is an inadequate basis for its pursuit. I argue on the contrary that an endless string of design examples is precisely at the core of how design research should operate, and that the role of theory should be to annotate those examples rather than replace them.

\section{THEORY IN RESEARCH THROUGH DESIGN}

Conceptual work appears in many forms as a routine part of research through design work. Whenever practitioners describe their influences, discuss the rationales for design decisions, and articulate their assessment of what they have made and its importance, they engage in a form of implicit conceptual work by highlighting important issues, dimensions of similarity, and criteria for choices and success. To the extent these conceptual statements are articulated in general terms and applied to multiple examples, they become recognisable as theories in their own right. For instance, Djajadiningrat and his colleagues [6] develop a rich account of the importance of embodied movement that is substantially informed from reflections about a series of designs they and their students have produced. They start from a conceptual perspective that values bodily movement, but it is through consideration of the designs their students have made that they elaborate this theory.

Design researchers often 'borrow' conceptual perspectives from other disciplines and discuss their applicability for design. Examples that are widespread in the design community include notions of affordances, context and situatedness [27] (cited in [31]). Borrowed theories (or concepts) are often used both to inspire new designs and to articulate existing ones. In doing so, the perspectives are usually translated for use by designers. For instance, when Wiltse and Stolterman [32] suggest that interaction design should draw on the sensibilities of architecture, they do not refer to physical spaces but rather the social spaces opened by computational media. Translation can ultimately give rise to new concepts. For instance, Overbeeke and Wensveen [23] describe how they originally drew inspiration from Gibson's ecological psychology (e.g. [15]), and particularly the notion of affordances, but adapted this to focus on emotional appeal under the new name of 'irresistables'.

Manifestos are a third form of theory often produced as a part of research through design practice. These go beyond theoretical treatments drawn from other disciplines or developed from reflection on practice to suggest certain approaches to design as both as desirable and productive of future practice $[13,14,16,30,36]$. For example, in introducing their notion of 'Reflective Design', Sengers and her colleagues [30] suggest that 'reflection on unconscious values embedded in computing and the practices that it supports can and should be a core principle of technology design'. Similarly, Gaver advocated a ludic approach to design as 'an antidote to assumptions that technology should provide clear, efficient solutions to practical problems' [13]. Typically, such manifestos will describe design practice to illustrate their approach, and borrow theories to justify it, but their primary function is to build an account of a practice to be pursued in the future.

Frameworks for design play a similar role to manifestos, but tend to downplay both their theoretical commitments and normative stance. For example, Forlizzi's Product Ecology [10] outlines a number of factors involved in designing for products to be used together and suggests methods to approach each stage. The framework is intended to allow 'flexible, design-centred research planning and opportunity seeking', and avoids prescribing appropriate methods. In characterising a class of design situations as it does, however, and in its ontology for describing the factors involved, the framework nevertheless implies a conceptual orientation, and can be considered a form of theoretical output from research through design.

A final genre of theory from research through design seeks to characterise research through design itself, and often to suggest normative standards for how research through design should be conducted, what should 'count' as research through design, and the appropriate standards for work done in this way. For instance, Zimmerman \& Forlizzi [34] 
argue that the HCI community will more easily accept contributions from research through design if it has an 'agreed upon form of practice, evaluation, and outcome' and suggest this should come through the development of 'extensible, systemic approaches' to theory development. Stolterman [31], in contrast, highlights the tendency for design to produce ultimate particulars, and suggests this poses problems for developing generalisable theories. Although these sorts of treatments can be considered as wide-ranging manifestos, this essay - which itself is intended as a contribution to meta-theory - addresses the more specific, content-oriented theories described earlier.

Arguably, designers in the HCI community have produced an ample amount of conceptual work arising from articulations of practice, borrowed ideas, manifestos and frameworks. But how good is this conceptual work? Should we be proud of the corpus of conceptualisations that have grown, or is there something inadequate about our achievements? To explore these questions, I compare the theories produced by research through design with several influential accounts of scientific theory.

\section{SCIENTIFIC AND DESIGN THEORIES}

In this section, I describe two contrasting accounts from the Philosophy of Science, primarily to shed light on the characteristics of research through design, but also to problematise the (potentially tacit) accounts of science that seem to underlie some views of research. Although other accounts of theory, for instance from the humanities, can also shed valuable light on design [2], there are several reasons why accounts of scientific theory are a useful foil against which to consider the conceptual work produced as a result of design practice. First, fields such as the Philosophy of Science and Science and Technology Studies (STS) offer sophisticated and well-developed discussions of the nature of theory and how it operates within a scientific context (see [5] for a valuable overview) which may serve as a fertile source of insight about theory in any field. In addition, one of the fundamental issues addressed within the Philosophy of Science concerns how to distinguish scientific fields from non-scientific ones (occasioned, for example, by arguments about evolution v. 'creation science'). These discussions highlight a number of issues that can be useful in considering the nature of design as a research endeavour, and the likely characteristics of theory likely to appear as a result of that endeavour.

\section{Falsifiability}

One influential account of science holds that 'the criterion of the scientific status of a theory is its falsifiability, or refutability, or testability' [24] (emphasis in the original). Popper proposed this criterion as a corrective to notions that scientific theories are primarily produced by induction, and thus are more powerful the more phenomena they agree with. As he notes, 'It is easy to obtain confirmations, or verifications, for nearly every theory - if we look for confirmations' ([24] p 7). But an endless number of confirmations cannot prove a theory. In fact, according to
Popper the probability that a particular theory is correct is zero no matter how much positive evidence is amassed (see [5] p. 70). In contrast, Popper suggests, a single incompatible result can disprove a theory. Feynman [8] makes a similar point in describing the search for a new physical law:

First we guess it. Then we compute the consequences of the guess to see what would be implied if this law that we guessed is right. Then we compare the result of the computation to nature, with experiment or experience, compare it directly with observation, to see if it works. If it disagrees with experiment it is wrong. In that simple statement is the key to science. [8] p. 156

From this perspective, the scientific process creates a kind of artificial evolution of theories: by eliminating weaker theories, only the strongest survive. Popper's point is that, for this to work, theories must be falsifiable in principle: "irrefutability is not a virtue of a theory (as people often think) but a vice' ([24], p. 7).

\section{Scientific Research Programmes}

Popper's emphasis on falsifiability, though influential in popular accounts of scientific reasoning, has been widely criticised as an inaccurate description of scientific practice. According to Kuhn [17], for example, 'normal science' involves 'puzzle-solving', in which researchers apply accepted theories to known problems or new domains. In normal circumstances, 'failure to solve a problem... is considered the fault of the scientist using the theory, not the fault of the theory itself' ([17] p 67).

Moreover, the idea that scientific theories are rejected when evidence contradicts them is historically inaccurate. Even new theories may not accord with data. Newton, for example, admitted when he published his gravitational theory that it could not account for the moon's orbit [3]. Feynman [8] describes how a new theory can win out over established evidence in recounting how the equations for 'weak decay' were established, in a passage striking for its inconsistency with his previous account:

...only the equation was guessed. The special difficulty this time was that the experiments were all wrong. How can you guess the right answer if, when you calculate the result, it disagrees with experiment? You need courage to say the experiments must be wrong. [8] p. 163.

How can scientists find the courage to champion theories over evidence? Lakatos [19] proposes an alternative account of scientific activity that emphasises scientific research programmes rather than individual theories. Programmes, according to his account, are characterised by a 'hard core' of theory, surrounded by a 'protective belt' of auxiliary hypotheses, and a 'powerful problem-solving mechanism' used to generate evidence and make it compatible with theory. In Lakatos' description, using these 
components a research programme 'digests anomolies and even turns them into positive evidence' ([19] p. 24). Programmes can change and evolve to adapt to new evidence, not by altering the 'hard core' of theory, but the 'protective belt' that surrounds it. For example, when Uranus was discovered not to move as predicted by Newtonian theory, rather than rejecting the theory, scientists predicted the discovery of the planet Neptune [5].

The ability for contradictory evidence to be turned into novel discoveries by research programmes is key to Lakatos' account. For him, research programmes do not succeed because they are unchallenged by disconfirming evidence. Instead, he writes:

...all the research programmes I admire have one characteristic in common. They all predict novel facts, facts which had been either undreamt of, or have indeed been contradicted by previous or rival programmes. ([19] p. 24).

Lakatos' account of scientific research programmes paints a very different picture of science from the Popperian one: instead of stressing scientific activity as a matter of trying to discredit theories, it emphasises scientific research as a dynamic machine for generating new knowledge, new understandings and new discoveries.

\section{Why Design Theory is Unfalsifiable}

Research through design is clearly unscientific if Popper's [24] criterion of falsifiablity is accepted. Theories are too vague, and practice is usually intended to confirm theories, not falsify them. To be sure, Popper's account is widely criticised both in Philosophy of Science and by practicing scientists. Still, it holds a potentially unflattering mirror up to the theories produced as a part of research through design. If confirmation cannot be used as proof, how do we validate them? Rather than seeing difficulties in validation as a problem for design theory, I would suggest this reveals two important characteristics of the conceptual work produced in association with research through design.

\section{Theory Underspecifies Design}

Design often addresses wicked problems [26] which are complex enough that no correct solutions exist a priori and for which formulating the situation is integral to addressing it. Moreover, design is an activity that involves many different decisions, dealing with many different and potentially independent factors of an artefact, all situated within the specific circumstances of production and use (c.f. [7]. Finally, is productive in the sense that it changes the context of its own activities [21]. In short, theory by necessity under-specifies design activities.

The implication of this underspecification is that the theories produced by research through design are not falsifiable in principle. Whether they build on borrowed theory or observation of the world or of specific design examples, such theories take the form designing for $X$ can lead to successful outcomes, where X may be 'the self' [36], 'homo ludens' [13], 'suppleness' [16], or 'simultaneous analogue control of multiple parameters' [6]. The problem is, the argument is not that $\mathrm{X}$ will always lead to successful designs (however success is evaluated), which would clearly be open to refutation. How could it be? There are too many other factors involved in a design project to make that kind of guarantee. Instead, there is always an implicit sometimes in statements about how to design successfully, to reflect the myriad of factors that remain untheorised yet crucial to a project's success. Assertions that $\mathrm{X}$ will sometimes lead to successful outcomes, however, are unfalsifiable, because no number of unsuccessful design efforts would actually disprove the assertion - after all, the next attempt might be successful.

Underspecification makes falsification difficult in practice as well as in principle. Even where potentially falsifiable assertions are offered, tests which are simultaneously unambiguous and ecologically valid are inconceivable. Consider, for example, a key claim made in a paper arguing for the benefits of ambiguity in design:

By impelling people to interpret situations for themselves, [ambiguity] encourages them to start grappling conceptually with systems and their contexts, and thus to establish deeper and more personal relations with the meanings offered by those systems. [14].

Although this is in principle a falsifiable statement, testing it in the Popperian sense would mean arranging a comparison in which no difference in conceptual grappling, or the depth and intimacy of personal relations to meaning, could be established between comparable ambiguous and unambiguous systems. The problem is in determining how to construct such a systems to be 'comparable'. Simply tweaking an unambiguous system to be ambiguous might address the assertion in a narrow sense, but it would be unrepresentative of the ways designers harness untheorised factors of a design to support its intended effects. In other words, the synthetic nature of design is incompatible with the controlled experiments useful for theory testing.

\section{Design Is Generative}

What I am arguing is that if designers were to change their practices to design for comparison or refutation, they would no longer be doing research through design. The notion of making falsifiable statements, or of arranging tests to refute such statements, runs against the grain of the methodological approach of research through design. Design, and research through design, is generative. Rather than making statements about what is, design is concerned with creating what might be, and moreover, in Zimmerman et al.'s [34] formulation, on making the 'right thing'.

The issue for designers is not to show that design theory can sometimes lead to bad designs. On the contrary, their concern is to sometimes create good ones. Similarly, the goal of conceptual work in research through design is not to develop theories that are never wrong, it is to create 
theories that are sometimes right. As Stolterman [31] puts it, 'designers can be prepared-for-action but not guided-inaction by detailed prescriptive procedures' (p 61). The generative nature of design has deeper implications for research through design, as I will argue later. For now, it is enough to note that, from Popper's perspective, one of the features that distinguishes design from science is its tendency to make generative statements rather than falsifiable ones.

Lakatos' characterisation of scientific research programmes, in contrast, paints a picture of theory seems more compatible with the nature of research through design. From this perspective, it is of little matter that conceptual approaches such as 'Reflective Design' [30] or 'Supple Interfaces' [16] or 'Designing for the Self' [36] are not falsifiable. Instead, what is at stake is their ability to inspire new designs. It is understandable why so many design manifestos, or examples of borrowed theory, are accompanied by examples of realised designs. What Popper would reject as 'merely' confirmatory evidence is, from this point of view, testimony to the fertility of the overarching theory. The difficulty of verifying design theory, at least through falsification, is not a flaw for research through design, as long as that theory can lead to productive research programmes.

\section{Theory from Research Through Design}

To be clear, I am not presenting Popper's and Lakatos' accounts as particularly representative of Philosophy of Science. In fact, by extracting these accounts from that complex and dynamic field, I wish in part to illustrate how unsettled and controversial accounts of science are. This lack of resolution, I would suggest, undermines attempts to characterise research through design (or design in general) either as a science or as a non-science. Personally, I tend to think that the nature of design practice and research is distinct from the ideals, and often practice, of science - but in the end, whether research through design can be considered a science depends on the account of science one adopts. Similarly, taking seriously the disagreements about the nature of science subverts any easy scientism in HCI more generally, because it destabilises the use of 'science' as shorthand for a set of practices and criteria assumed to be desirable. It is difficult to call for research to be more scientific if what it means to be scientific is under question (see [29]).

Instead, what this discussion suggests is the desirability of clearly articulating the methodological and conceptual features of current research through design practice, as well as the standards one might want to advocate for them. Here I have suggested that the theory produced from design practice tends to underspecify practice and to be generative in nature, and thus that it is provisional, contingent, and aspirational. These are all features that limit the potential 'extensibility and verifiability' of design theory, seen by some as possible and desirable. Instead, as I discuss in the remainder of this paper, research through design should be appreciated for its proliferation of new realities, and its theory considered as annotation of the artefacts that are its fundamental achievement.

\section{CONVERGENCE IN RESEARCH THROUGH DESIGN}

Calls for disciplinary reform in research through design appeal for more agreement about the 'methods, approaches, and outcomes' in the field. Greater integration would make assessment of new contributions fairer and more rigorous. Moreover, if we could all agree about the proper approaches, methods and outcomes to use, we could build upon one another's research in a cumulative fashion. Currently, however, there must be dozens of different 'manifestos' for design, each seeking to establish a research programme, and each with its own configuration of goals, issues, methods and realised exemplars. Why is there not more convergence in the field?

Convergence in the natural sciences is a given. Lakatos' research programmes, for example, describe scientific research in terms of historical movements in which researchers share a common set of practices and understandings. A similar description is developed by Kuhn [17] in his seminal account of 'scientific revolutions'. According to Kuhn, the reason 'normal science' can proceed is because researchers share a common paradigm, which, in Kuhn's usage, sometimes means an exemplar of successful research, and sometimes a set of common understandings about how to describe relevant phenomena, relevant issues, acceptable methodologies and outputs. Both accounts describe scientific research as convergent.

Even more striking, however, Lakatos' and Kuhn's accounts both suggest that, as a normal state of affairs, only one programme or paradigm will dominate a particular scientific field at any one time. Aristotelian physics gives way to Newtonian physics, which gives way in its turn to Relativity. For Kuhn, paradigm shifts occur when tension grows about a given paradigm's inability to resolve anomalies, a new possible view arises which solves those anomalies (or makes them irrelevant) and scientists choose to join the new paradigm. Because paradigms involve different 'world views', including ontologies, philosophies, methodologies, etc., choosing between two candidates cannot be done on rational grounds, according to Kuhn. Lakatos objects to this, suggesting the relative productivity of competing programmes as the grounds for scientists' choice amongst them. Both agree, however, that multiple programmes, or paradigms, cannot co-exist over time.

Why do multiple research programmes coexist in design? In the following sections I explore three possibilities.

\section{Design as Pre-paradigmatic Research}

According to Kuhn [17], the beginning of a scientific study of a given field comes with the widespread adoption of a single paradigm for its study. Until that time, research is characterised by competition amongst a number of different schools of thought, usually drawing on different philosophical foundations and orienting towards the field in 
different ways. Valuable contributions may be made by disparate approaches, but without shared assumptions about the correct way to approach a field, individual researchers must establish the rationale behind their approach from the very basics every time they seek to make a contribution. Because there are no agreed standards of importance, there is little sense of priority or order to the findings that are amassed. Progress is slow and in Kuhn's view does not really add up to a science.

When a particularly influential body of research is finally established, usually in the form of a theory that ties together multiple troublesome phenomena and suggests new paths for research, everything changes. Researchers no longer need to justify the basics of their research, but can concentrate on increasingly detailed and exacting contributions. They become more confident about devoting resources on studies which are difficult and timeconsuming, since they know they will be understood and valued by their fellow researchers. In general, working together on the issues suggested by a given paradigm, and using methods and techniques suggested by it, allows science to make significant progress. Pre-paradigmatic research, in contrast, must founder.

It is clearly possible to recognise the current state of research through design in this description of affairs. There is a proliferation of research programmes, and little agreement about the values for which we should design, the appropriate methods for doing so, standards for evaluation or agreed forms of output. Methods and techniques proliferate, and when they are taken up by others it is often without their underlying methodological orientation. Even what it means to undertake research as a form of design is subject to disagreement, with some suggesting that design projects should be constructed, in part, to explicitly test and produce theory $[33,35]$, while others (including myself) believe that theory should be allowed to emerge from situated design practice.

From this perspective, research through design lacks a shared paradigm. Without it, we cannot join forces around agreed issues, or find common standards for work we agree is solid enough to build upon: research through design cannot make progress. This is a tempting conclusion, one seemingly behind recent calls for formalism and standardisation $[9,35]$. Two other accounts, however, may also explain the seeming disunity of research through design when compared to research in the natural sciences.

\section{The Invisibility of Consensus}

One alternative account to that of the supposed preparadigmatic nature of research through design would suggest that the balance of agreement and controversy is not so different in research through design than it is in many fields of scientific research. This interpretation hinges on the observation that consensus is usually easier to see from a distance than it is to experience from within. After all, as Kuhn [17] suggests, researchers do not need continually to restate the agreed fundamentals of their field.
Instead their discourse tends to focus on controversies and debates around new findings and unresolved anomalies. From this perspective, scientific discourse may be characterised by far more controversy than is implied by Kuhn's account of normal, paradigm-driven research, and there may be more of a shared sense of understanding guiding research through design, than sometimes seems apparent.

If the natural sciences appear unified to an extraordinary degree, able to build upon a solid foundation of agreed knowledge, this may be a function of their presentation in the popular media, particularly with the advantage of hindsight. In contrast to Kuhn's characterisation of normal science as consisting of 'puzzle solving', other researchers paint a less cozy picture of scientific discourse. Bruno Latour [20], for instance, characterises scientific activity in terms of controversy in his description of the networks, instruments, inscriptions and theories deployed by scientists to gain acceptance for their ideas. Accounts such as this suggest that, although scientific work may be based on many fundamental agreements about how that work should be conducted, as Lakatos [19] and Kuhn [17, 18] suggest, the day-to-day scientific discourse may well exhibit just as much uncertainty, interpretation and debate as does research through design.

Conversely, research through design may come much closer to comprising a research programme, or sharing a paradigm (or set of paradigms) than we appreciate. After all, most of us working in research through design seem to share a set of common values. For instance, most pursue some variation on user-centred design, agreeing that some contact with the potential audiences for the things we make is desirable before, during or after design work itself. Most of us assume that exploring a wide space of potential designs, whether through sketching, scenarios, narratives or design proposals, is crucial in achieving a good outcome. Most of us appreciate the value of craft and detail in our work. Most fundamentally, most of us agree that the practice of making is a route to discovery, and that the synthetic nature of design allows for richer and more situated understandings than those produced through more analytic means. Perhaps we do not all agree with all of these assumptions all of the time, and perhaps it is endemic to design that all assumptions should be tested, but there certainly seems to be the outlines of a broad consensus underlying research through design.

Again, the shared assumptions underlying research through design practice are precisely those unlikely to be addressed in the literature. It is the speculative ideas, the novel, and the disagreements that we are most likely to discuss. This may lead us to underestimate the discord of science, however, and to overestimate the divergence of research through design. From this point of view, calls for standardisation, formalisation, overarching theory and the like are misplaced; we already share many of the attributes 
of a research paradigm, and seeking to reduce diversity its cutting edge will just inhibit progress.

\section{The Many Worlds of Design}

There is yet another possible interpretation for the apparent lack of convergence in research through design, which sidesteps the opposing views that we either need a shared paradigm to make progress, or already have a shared paradigm and need to recognise controversy as a sign of progress. In this view, whether or not research through design is built on certain assumptions about its conduct, it will inevitably be characterised by greater diversity and less convergence than the natural sciences because of the inherent nature of its field of study.

Many other fields lack the convergence seen over time in the natural sciences. For instance, Kuhn explains that his account of the paradigmatic nature of the natural sciences was inspired in part by the continual disagreements he witnessed amongst social scientists ([17]; c.f. [3]). Multiple schools co-exist in the arts, architecture, fashion and product design. In all these fields, controversies are seldom settled in such a way that new work can build on accepted results, the way science seems to do. Instead, new artistic movements overturn the basic values of their predecessors, hemlines go up and down, and even the most basic of household fittings are the subject of continual redesign and rethinking. These disciplines do not converge as the sciences do; instead they are cumulative in the way a conversation is, elaborating on what has gone before, but seldom aiming for or finding resolution. Why do disciplines such as these fail to show the convergence of science?

For the natural sciences, there is a strong presumption that the object of study is a single, unitary world that pre-exists and is independent of its observers. This core belief seems naturally to lead to convergence, simply because, if there are two incompatible accounts of the same physical domain, one must be better than the other (see [18] for one definition of 'better'). Research and practice in design and the arts, in contrast, do not describe a single, independent world, they are generative, investigating how to create new ones. Debates may rage about 'better' ways to go about this, but these cannot refer to evidence from a single, independent world as an ally (c.f. [20]). Multiple, incompatible worlds co-exist routinely for these fields.

Moreover, design and the arts change the context in which they operate. When the original iPad was designed, for instance, tablet computers were not widely known or available. Now anybody seeking to research or develop tablet computers - or anything at all, for that matter - is designing for a different world, one in which the iPad exists. One of the implications of the way generative disciplines change the context in which they work is that this sets the conditions for a feedback loop in which the development (and adoption) of a new design sets the scene for the development of variations, accessories, applications and reactions: whole new areas of reality. Another implication is that development in these fields does not just take the form of accumulation of incontrovertible results, but of reactions, reconsiderations and fresh beginnings.

Social sciences, too, change the world in which they operate. Osborne and Rose [22] for example, describe how the development of public opinion research gave rise to public opinion itself, as something to be identified, measured, and considered in policy formation and product development. Law and Urry [21] observe that labelling theory, as proposed by deviance theorists, led to policies of de-institutionalisation, and suggest that by bringing social inequality into view, British sociologists gave rise to the policies intended to address it. The social world, in this view, is not the subject of objective, independent observation. Instead:

"...different research practices might be making multiple worlds, and that such worlds might be equally valid, equally true, but simply unlike one another.' [21] p. 397.

If research is performative in this way, they suggest, then the research is no longer concerned primarily with epistemology (how we know about our object of study) but also involves 'ontological politics', a concern with what is being made.

From this point of view, the reason that research through design is not convergent is that it is a generative discipline, able to create multiple new worlds rather than describing a single existing one. Its practitioners may share many assumptions about how to pursue it, but equally, they may build as many incompatible worlds as they wish to live in. We may wish to improve the standards of research within the field, but from this perspective we should realise that what we mean by 'improve', what criteria we propose, even the assumption that shared standards are necessary, possible or desirable, are potentially repressive acts of ontological politics.

\section{THEORY AS ANNOTATION}

What is an appropriate role for theory in research through design? On the one hand, it is plausible to argue that if design is to contribute to HCI research, we should turn greater attention to theory-making both as a way of capturing and communicating new learning to the research community and as a way of guiding design practice [33, 35]. Most practicing designers, however, do not engage with major theoretical approaches in HCI. Instead, they use a more eclectic mix of design techniques and orienting concepts ([27] cited in [31]). In addition, designers often turn to existing examples of design to inform the development of their own ideas. Why might this be so? I suggest it is both because of the provisional nature of theory, and the definite nature of designed artefacts.

As I have suggested, a great deal of design theory tends to be generative and suggestive, rather than verifiable through falsification. This seems self-evident in the case of 
'manifestos', but also of more grounded generalisations from particular design examples (e.g. [6]). The problem here is not just that theory underspecifies design, so that practitioners will be faced by innumerable decisions whatever theory they use, but that theory is underspecified by design, in the sense that many aspects of a successful design will not be captured by a given theory. Thus in order to understand accounts of how to achieve simultaneous control over multiple functions successfully, for example, it is useful to consider specific designs such as a rotary controller for a microwave oven [6].

Design examples are indispensible to design theory because artefacts embody the myriad choices made by their designers with a definiteness and level of detail that would be difficult or impossible to attain in a written (or diagrammatic) account. As Stolterman [31] suggests, design is concerned with the 'ultimate particular', a concept, he suggests, that has 'the same dignity and importance as truth in science' ([31] p. 59). Theories may be provisional, but designed artefacts (as opposed to demonstrators, for example) are not. As Carroll and Kellogg [3] have argued, a designed artifact is a 'theory nexus': the choices made by designers reveal both the issues they think are important, and their beliefs about the right way to address those issues. The implicit theories embodied in objects, from this perspective, range from the philosophical (what values should designs serve?) to the functional (how should those values be achieved in interaction) to the social (what will the people who use this be like?) to the aesthetic (what form and appearance is appropriate for the context?). Moreover, artefacts do not address these issues analytically, but represent the designer's best judgement about how to address the particular configuration of issues in question.

Another metaphor for much the same point is that designs can be seen as occupying a point in design space, or perhaps more accurately creating a design space around themselves (see [12]). In this account, designers can explore the implications of a given design by moving around the point it inhabits to explore new design possibilities, or even by jumping away from it along understood dimensions, whether to apply some of the same decisions to new domains or to react against them.

If artefacts embody theory, however, they do not encode it, and if they occupy a point in a design space, they do not highlight the salient or fruitful dimensions of variation that space offers. One of the valuable roles of design theory, from this perspective, is in making accessible the kinds of decisions and rationales that comprise an artefact's embodied theory, or give dimensionality to its design space. In this case, however, then instead of theories predominating, with design examples serving as mere illustrations, design theory is best considered a form of annotation, serving to explain and point to features of 'ultimate particulars', the truths of design.

\section{Annotated Portfolios}

Beyond single artefacts, however, annotated portfolios may serve an even more valuable role as an alternative to more formalised theory in conceptual development and practical guidance for design ${ }^{1}$. If a single design occupies a point in design space, a collection of designs by the same or associated designers - a portfolio - establishes an area in that space. Comparing different individual items can make clear a domain of design, its relevant dimensions, and the designer's opinion about the relevant places and configurations to adopt on those dimensions (c.f. [7]). To put it another way, multiple examples can start to tease the individual concerns and judgements involved in a single situated design out of the particular configuration to which they were applied, making clear both the dimensions along which a designer's choices may range and the invariances among them.

As artefacts are to theory, from this perspective, design portfolios are to research programmes . For instance, Dieter Rams' work for Braun and Vitsoe has been characterised as 'defining an elegant, legible, yet rigorous visual language for its products' (www.designmuseum.org/design/dieterrams), but to appreciate what this might mean one must turn to a portfolio of his work (Figure 1). Comparing the variety of stereo equipment, kitchenware and grooming appliances that he designed, one begins to appreciate the qualities that hold across the examples, and those that are more particular to specific designs. Rams offered a particular form of conceptual writing to help with this, defining his approach to 'good design' in ten concise principles (e.g. 'good design is unobtrusive', 'good design is thorough down to the last detail') accompanied by short explanations. These annotate the portfolio, drawing attention to important features and to salient details that might otherwise be overlooked. The passages may seem too terse to serve as substantial conceptual contributions by themselves, but in the presence of the portfolio, they give a strong sense of Rams' style and philosophy. One can imagine 'designing in the style of Dieter Rams' without the need for a detailed formal theory to direct decisions.

The notion of annotated portfolios is not a formal one. What is defining to the concept is not how materials are presented, but that a balance is achieved between descriptions of specific, detailed examples of design practice, and articulations of the issues, values and themes which characterise the relations among the collection, and to which the examples suggest answers. The appropriate presentation of an annotated portfolio may vary depending on material, purpose and audience. For instance, the detailed annotations shown in Figure 1 are extremely compact and focus more on highlighting features of the artefacts than on elaborating the overarching conceptual themes. A different presentation of Rams' work, at

\footnotetext{
${ }^{1}$ Many of the ideas here about annotated portfolios have been developed in collaboration with John Bowers.
} 

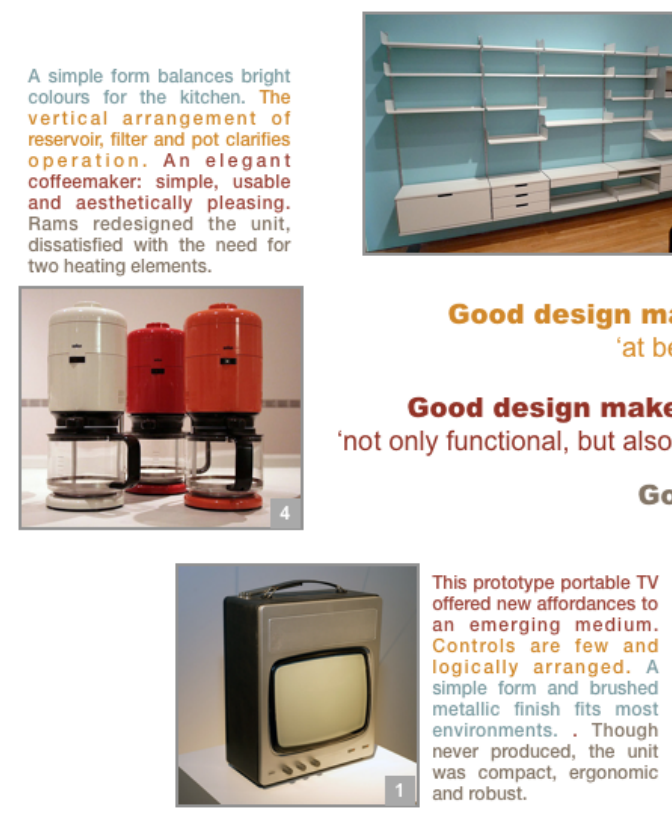

(Images from flickr.com's Creative Commons. Credits: 1 Nick Wade, 2 Rene Spitz, 3 Brett Wayn, 4 '•')

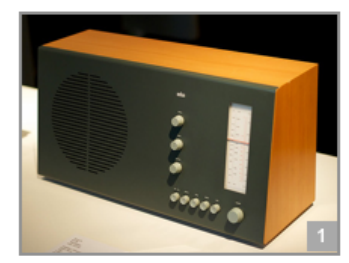

Not only is it clear how to use this adio, but its mixed material brings technology into domestic according to different groups of functions. The slate grey fascia fades into the environment. The radio's details support its functions and aesthetios wooden enclosure.

\section{Good design makes a product understandable}

'at best, it is self explanatory'

Good design makes a product useful

'not only functional, but also psychological and aesthetic'

Good design is unobtrusive 'both neutral and restrained'

Good design is thorough, down to the last detail 'nothing must be arbitrary or left to chance'
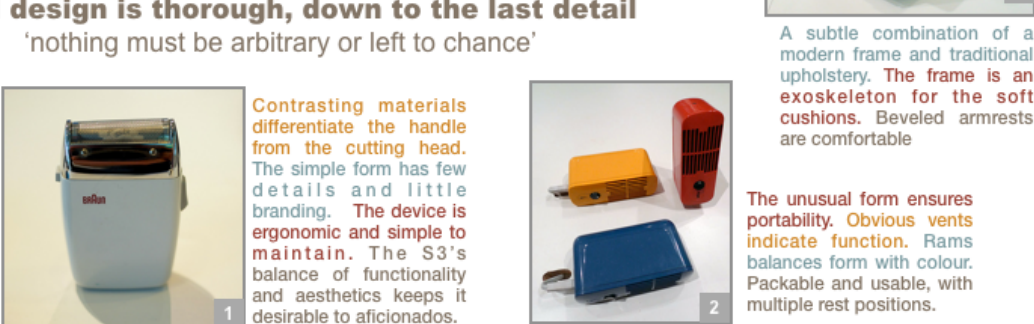
A subtle combination of a upholstery. The frame is an
exoskeleton for the soft cushions. Beveled armrests are comfortable

The unusual form ensures portability. Obvious vents indicate function. Rams balances form with colour Packable and usable, with ultiple rest positions.

Figure 1. Sketch design for an annotated portfolio of Dieter Rams' designs for Braun and Vitsoe (note that annotated portfolios are not defined by their graphic presentation)

www.vitsoe.com, emphasises his design principles over the portfolio of products themselves. A more detailed examination of Rams' philosophy might well take the form of an illustrated essay. What links all these presentations is the mutually informative juxtaposition of conceptual annotations with specific design examples, in such a way that neither dominates, and neither is subservient.

Annotated portfolios are, in several respects, the converse of Alexander's [1] design patterns. They are not intended to abstract regularities from repeated attempts to design for the same domains. Instead, they maintain the particularity of individual examples, while articulating the ideas and issues that join and differentiate them. Juxtaposing designs with annotations supports appreciation of the conceptual dimensions of designs on the one hand, and, by yoking them to particular design manifestations, grounds and specifies theoretical concepts on the other. Portfolios can support multiple conceptual perspectives, and similar perspectives can be applied to different portfolios, reflecting the lack of convergence in the field as a virtue. Most fundamentally, annotated portfolios respect the 'ultimate particular' of the designed artefact, rather than abstracting across instances as pattern languages do, while allowing for the 'extensibility and verifiability' for which some of the HCI design community have called.

I am not proposing here that annotated portfolios subsume all other forms of design theory. Theoretical writing remains important in articulating the issues, rationales, and lessons that are embodied by design, particularly for an interdisciplinary audience. I am suggesting, however, that we develop annotated portfolios as a serious form of theoretical contribution appropriate to research through design. More fundamentally, I am suggesting that, however valuable generalised theories may be, their role is limited to inspiration and annotation. It is the artefacts we create that are the definite facts of research through design.

\section{CONCLUSION}

In this essay, I have explored some of the issues that complicate calls for disciplinary consolidation in the HCI research through design community.

First, I suggest that we should moderate expectations of creating extensible and verifiable theory. Comparisons with accounts from the Philosophy of Science indicate both how provisional, contingent and aspirational design theories tend to be, but also how such conceptual work may nonetheless inspire thriving research programmes. In addition, continuing controversies about how to characterise science should help undermine assumptions about research that draw on HCI's tendencies towards scientism. Finally, they also indicate the futility of debating whether design is or should be a science. Rather than worrying about accepting or rejecting some ideal version of 'science', I suggest, we should reflect on the appropriate ways to pursue our research on its own terms.

Second, I suggest that attempts to establish disciplinary norms of process or outcome are political acts to be approached with care. Considering possible accounts of a lack of convergence in research through design suggests that greater consensus may be emerging in research through design than is sometimes acknowledged. At the same time, convergence may not be the only or best model for 
progress. Research through design may develop not only through increasing agreement, but also through discursiveness and elaboration. We may build on one another's results, but we can also usefully subvert them, suggest alternatives, or establish entirely new constructions, and this applies equally to our concepts, methods, processes, artefacts and approaches to evaluation. From this perspective, consensus can come to look like constraint.

Finally, I have advocated the recognition of annotated portfolios as a way of reflecting and valuing the particular nature of design theory and practice. These, I suggest, allow design theory's provisionality, specificity and diversity to be turned to advantage through grounding in specific sets of detailed design examples. They may provide a way to further our ambitions to produce relevant and rigorous theoretical work, while allowing multiple perspectives to flourish. Best of all, emphasising theory as the annotation of portfolios offers respect to the endless string of design examples that should remain at the heart of our work.

\section{ACKNOWLEDGEMENTS}

This research was supported by the European Research Council's Advanced Investigator Award no. 226528, 'ThirdWaveHCI'. Many thanks go to Phoebe Sengers, Anne Schlottmann and especially John Bowers for comments and suggestions. I am also grateful to reviewers for their thoughtful critiques, particularly the anonymous $\mathrm{R} 4$, who recognised that this essay addresses, in part, 'an elephant in the room...: the move towards "scientism" in HCI'.

\section{REFERENCES}

1. Alexander C, Ishikawa S (1977) A pattern language: towns, buildings, construction. Oxford University Press.

2. Bardzell J, Bardzell S, DiSalvo C, Gaver B and Sengers P (2012). Panel: The humanities and/in HCI. CHI'2012.

3. Collins R (1994). Why the Social Sciences Won't Become High-Consensus, Rapid-Discovery Science. Sociological Forum 9(2) 155 - 177.

4. Crampton-Smith, G. and Tabor, P. 1996. The role of the artistdesigner. In Bringing Design To Software, T. Winograd, Ed. ACM, New York, NY, 37-61.

5. Curd M and Cover J (1998). Philosophy of Science: The Central Issues. London: WW. Norton \& Co.

6. Djajadiningrat T, Matthews B, Stienstra M. (2007). Easy doesn't do it: skill and expression in tangible aesthetics. Pers Ubiquit Comput 11:657-676.

7. Fallmann D (2003). Design-oriented human-computer interaction. CHI'2003, 225-232.

8. Feynman, R. (1989). The character of physical law. London: MIT Press.

9. Forlizzi J, DiSalvo C, Bardzell J, Koskinen I, and Wensveen S. (2011). Quality control: A panel on the critique and criticism of design research. CHI 2011 Panel, 823 - 826.

10. Forlizzi, J. (2008). The Product Ecology: understanding social product use and supporting design culture. International Journal of Design 2 (1), 11 - 20.

11. Frayling, C. Research in Art and Design. Royal College of Art Research Papers 1, 1 (1993),1-5.

12. Gaver W. (2011) Making spaces: how design workbooks work. Proc. CHI '11, 1551-1560.
13. Gaver, W., (2009). Designing for Homo Ludens, Still. In (Re)searching the Digital Bauhaus. Binder, T., Löwgren, J., and Malmborg, L. (eds.). London: Springer, pp. 163-178.

14. Gaver, W., Beaver, J., and Benford, S. (2003). Ambiguity as a resource for design. Proc. CHI'03, Ft. Lauderdale. New York: ACM Press.

15. Gibson J. (1979). The ecological approach to visual perception. Boston: Haughton Mifflin, 1979.

16. Isbister K, and Höök K. (2007). Supple interfaces: designing and evaluating for richer human connections and experiences. Proc CHI'07 extended abstracts, 2853 - 2856.

17. Kuhn, T (1970). The Structure of Scientific Revolutions. London: University of Chicago Press.

18. Kuhn, T (1977). Objectivity, value judgment, and theory choice. Reprinted in [3].

19. Lakatos I (1977). Science and pseudoscience. Reprinted in [3].

20. Latour, B. (1987). Science in Action. Cambridge, Mass: Harvard University Press.

21. Law, J. and Urry, J. (2004). Enacting the social. Economy and Society 33(3) pp 390-410.

22. Osborne, T. and Rose, N. (1999). Do the social sciences create phenomena?: the example of public opinion research. British Journal of Sociology 50(3) pp 367 - 396.

23. Overbeeke K and Wesveen, S. (2003). From perception to experience, from affordances to irresistibles. Proc. DPPI'03, $92-97$.

24. Popper K (1963). Science: conjectures and refutations. Reprinted in [3].

25. Pullin, Graham (2010) Curating and creating design collections. Design and Culture 2 (1), 309-328.

26. Rittel H, and Webber M. (1974). Dilemmas in general theory of planning. Design Research and Methods, 8(1), 31 - 39.

27. Rogers, Y. (2004). New theoretical approaches for humancomputer interaction. In B. Cronin (Vol. Ed.), Annual review of information, science and technology: Vol. 38 (pp. 87 - 143). Medford, NJ: Information Today.

28. Schoen, D (1983). The reflective practitioner, how professionals think. New York, Basic Books.

29. Sengers P (2006). Must design become 'scientific'? DIS'06 Workshop on Exploring Design as a Research Activity.

30. Sengers P, Boehner K, David S, and Kaye J. (2005). Reflective design. Proc. AARHUS'05, 49 - 58. 24

31. Stolterman, E. (2008). The nature of design practice and implications for interaction design research. International Journal of Design 2(1), 55 - 65.

32. Wiltse, H. and Stolterman, E. (2010). Architectures of interaction: An architectural perspective on digital experience. Proc. NordiCHI 2010, 821 - 824.

33. Zimmerman J and Forlizzi J. (2008) The Role of Design Artifacts in Design Theory Construction. Human-Computer Interaction Institute. Paper 37.

34. Zimmerman J, Forlizzi J, and Evenson, S. (2007). Research through design as a method for interaction design research in HCI. Proc. CHI'07 493-502.

35. Zimmerman J, Stolterman E, and Forlizzi J (2010). An analysis and critique of research through design: toward a formalization of a research approach. DIS 2010, 310 - 319.

36. Zimmerman, J. (2009). Designing for the Self: Making products that help people become the person they desire to be. Proc. CHI 2009, 395 - 404. 